\title{
Biomaterials-assisted spheroid engineering for regenerative therapy
}

\author{
Na-Hyun Lee ${ }^{1,2}$, Oyunchimeg Bayaraa ${ }^{1,2}$, Zhou Zechu ${ }^{1,2}$ \& Hye Sung Kim ${ }^{1,3, *}$ \\ ${ }^{1}$ Institute of Tissue Regeneration Engineering (ITREN), Dankook University, Cheonan 31116, ${ }^{2}$ Department of Nanobiomedical Science \\ and BK21 NBM Global Research Center for Regenerative Medicine, Dankook University, Cheonan 31116, ${ }^{3}$ Department of Regenerative \\ Dental Medicine, College of Dentistry, Dankook University, Cheonan 31116, Korea
}

Cell-based therapy is a promising approach in the field of regenerative medicine. As cells are formed into spheroids, their survival, functions, and engraftment in the transplanted site are significantly improved compared to single cell transplantation. To improve the therapeutic effect of cell spheroids even further, various biomaterials (e.g., nano- or microparticles, fibers, and hydrogels) have been developed for spheroid engineering. These biomaterials not only can control the overall spheroid formation (e.g., size, shape, aggregation speed, and degree of compaction), but also can regulate cell-to-cell and cell-to-matrix interactions in spheroids. Therefore, cell spheroids in synergy with biomaterials have recently emerged for cell-based regenerative therapy. Biomaterials-assisted spheroid engineering has been extensively studied for regeneration of bone or/and cartilage defects, critical limb ischemia, and myocardial infarction. Furthermore, it has been expanded to pancreas islets and hair follicle transplantation. This paper comprehensively reviews biomaterials-assisted spheroid engineering for regenerative therapy. [BMB Reports 2021; 54(7): 356-367]

\section{INTRODUCTION}

Cell-based therapy is a promising approach for tissue regeneration. To fill tissue loss and restore tissue functions of injured tissues, a major cell type of the tissue to be regenerated is often chosen as a cell source for transplantation. For example, chondrocytes are used for cartilage defect repair and cardiomyocytes are used for myocardial infarction repair. Among many cell sources, mesenchymal stem cells (MSCs) are the most clinically relevant and frequently used cell type for

*Corresponding author. Tel: +82-41-550-3075; Fax: +82-41-559-7839; E-mail: hskim1213@dankook.ac.kr

https://doi.org/10.5483/BMBRep.2021.54.7.059

Received 20 April 2021, Revised 23 May 2021, Accepted 15 June 2021

Keywords: Biomaterial, Regenerative medicine, Spheroid, Spheroid engineering, Tissue regeneration regenerative therapy due to their self-renewal ability, multipotency, low immunogenicity, and secretion of regenerative paracrine factors (1-3). MSCs-based regenerative therapy has been clinically demonstrated to have versatile therapeutic potential. However, its clinical outcome is not satisfying due to poor survival and engraftment of transplanted MSCs in vivo (4). To compensate the low efficiency of cell-based therapy, a large number of cells are required for transplantation (5-7). Cells are generally expanded in a two-dimensional (2D) monolayer culture system using a conventional culture method. However, a monolayer culture system with flattened nucleus and cytosol, a lack of cell-to-cell interaction, and a loss of cell-to-matrix interaction during the cell isolation procedure does not represent the three-dimensional (3D) cellular microenvironment in the body (8). Especially, cells require both cell-to-cell and cellto-matrix interactions for their survival and proliferation. For example, primary articular chondrocytes and hepatocytes can rapidly lose their original phenotypes and functions when they are cultured in a monolayer system $(9,10)$. This can compromise therapeutic effects of cells transplanted in vivo. Thus, $3 \mathrm{D}$ cell culture systems as alternatives have been developed to overcome limitations of conventional monolayer cell culture systems (11).

In the body, cells are constantly interacting with their surrounding extracellular matrix (ECM) and adjuvant cells to maintain their homeostasis and functions according to physicochemical cues from the surroundings (12). Thus, 3D cell culture systems should provide a physicochemical microenvironment mimicking the in vivo cell surroundings (13). A spheroid culture method is one of 3D cell culture systems that is easily reproducible and highly reliable for generating 3D cell-to-cell interactions (13). Cells are aggregated into a spheroid and cultured in suspension. Various spheroid fabrication methods such as hanging drop, non-adherent culture plate, gel embedding, magnetic levitation, and spinner culture have been reported $(11,14-16)$. It has been demonstrated that cell spheroids exhibit improved cellular functions in vitro as compared to a monolayer culture (17). More importantly, in vivo therapeutic effects of cell spheroids are superior to single cell transplantation due to their greater survival and engraftment capacities in harsh conditions $(18,19)$. Despite these benefits,

ISSN: 1976-670X (electronic edition)

Copyright (C) 2021 by the The Korean Society for Biochemistry and Molecular Biology

(c) This is an open-access article distributed under the terms of the Creative Commons Attribution Non-Commercial License (http://creativecommons.org/licenses/by-nc/4.0) which permits unrestricted non-commercial use, distribution, and reproduction in any medium, provided the original work is properly cited. 
cell spheroids often face issues associated with necrotic cores due to the lack of oxygen transportation (14). Therefore, biomaterials-assisted spheroid engineering has been extensively developed for successful regenerative therapy (14). A combination of culture/implantation with biomaterials such as nano- or microparticles, fibers, and hydrogels not only can improve the survival of spheroids in vivo, but also can protect spheroids in harsh conditions where they are implanted, leading to improved therapeutic effects of transplanted cells $(20,21)$.

In this review, we mainly focus on spheroid-based tissue regenerations of bone defects, cartilage defects, critical limb ischemia, cardiac defects, pancreas and hair follicles in which spheroid formation/configuration is essential for cell transplantation. We also discuss specific strategies of spheroid engineering combined with advanced biomaterials for targeting each tissue.

\section{SPHEROID FORMATION METHODS}

Unlike a 2D monolayer culture system, 3D spheroid culture

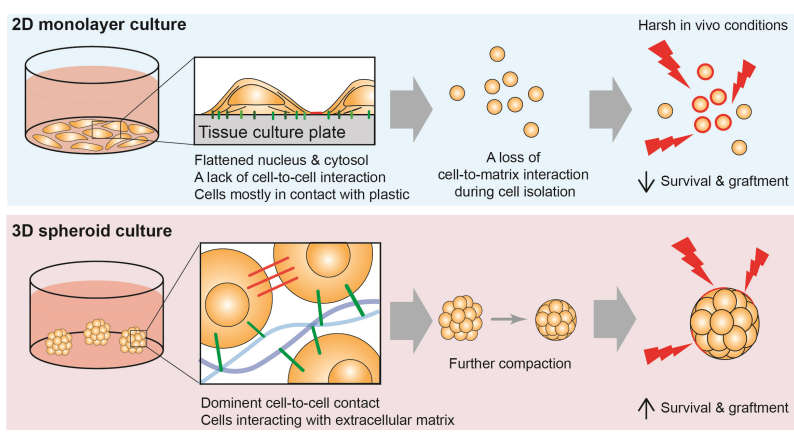

Fig. 1. Differences between $2 \mathrm{D}$ monolayer culture and $3 \mathrm{D}$ spheroid culture for potential clinical applications. systems can create a more in vivo-like microenvironment (Fig. 1). A 2D monolayer cell culture on tissue culture plates lacks cell-to-cell interaction and cells are mostly in contact with plastic. Cytoskeletal actin of cells cultured on tissue culture plates are highly polarized and thus nuclear flattening and epigenetic changes $(8,22)$. Furthermore, cells lose cell-to-cell and cell-to-matrix interaction during cell isolation, which worsens the cell survival and engraftment in vivo. On the other hand, in 3D spheroids, cell-to-cell contacts are dominant, and cells also interact with extracellular matrix with non-apical morphology. It has been demonstrated that spheroids formation enhances survival and engraftment of transplanted cells in vivo and increases growth factor secretion and target tissue integration $(23,24)$

There are various methods including microwells, hangingdrop, microencapsulation, centrifugation, magnetic levitation and spinning/rotating for spheroid formation (Fig. 2). In principle, the methods increase cell-to-cell cohesion by inducing spontaneous cellular assembly, applying physical forces (e.g. gravitational, centrifugal, or magnetic forces) or confinement (e.g. micro-structured surfaces or microcapsules) (Table 1). However, most of methods involve labor-intensive process, low yield,

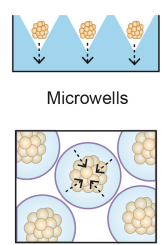

Microencapsulation

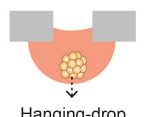

Hanging-drop

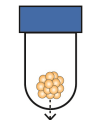

Centrifugation

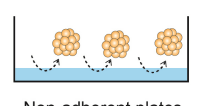

Non-adherent plates

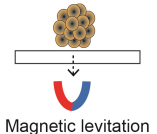

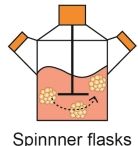

Spinnner flasks

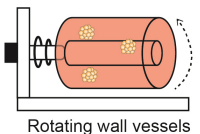

Fig. 2. Methods of spheroid formation.

Table 1. Methods for spheroid formation

\begin{tabular}{|c|c|c|c|c|}
\hline Method & How to & Driving force & Advantages & Disadvantages \\
\hline Hanging drop & $\begin{array}{l}\text { Make droplets of cell suspensions on a } \\
\text { lid of tissue culture plate, the lid is } \\
\text { flipped upside-down, and culture in } \\
\text { a humid condition }\end{array}$ & $\begin{array}{l}\text { Gravitational forces and } \\
\text { physical confinement }\end{array}$ & Easy to control spheroid size & $\begin{array}{l}\text { A limited volume of } \\
\text { droplets }(<50 \mu \mathrm{l}) \\
\text { Difficulty in changing } \\
\text { culture medium }\end{array}$ \\
\hline Microwells & $\begin{array}{l}\text { Confine cells in physical compartments } \\
\text { at a micrometer scale }\end{array}$ & $\begin{array}{l}\text { Gravitational forces and } \\
\text { physical confinement }\end{array}$ & & A difficulty in harvest \\
\hline Centrifugation & $\begin{array}{l}\text { Force cells aggregate at the bottom of } \\
\text { a centrifuge tube }\end{array}$ & Centrifugal forces & & $\begin{array}{l}\text { Cellular damages by } \\
\text { excessive external } \\
\text { forces }\end{array}$ \\
\hline Magnetic levitation & $\begin{array}{l}\text { Force magnetized cells form into } \\
\text { spheroids }\end{array}$ & Magnetic force & & $\begin{array}{l}\text { Cytotoxicity of } \\
\text { magnetic materials }\end{array}$ \\
\hline Microencapsulation & Confine cells into microcapsules & Physical confinement & & Batch variations \\
\hline Non-adherent plates & $\begin{array}{l}\text { Interrupt cell adhesion to the plates, } \\
\text { make cells rather aggregate to each } \\
\text { other }\end{array}$ & Spontaneous aggregation & $\begin{array}{l}\text { One step spheroid formation and } \\
\text { suspension culture } \\
\text { Less labor intensive }\end{array}$ & $\begin{array}{l}\text { Low yield and various } \\
\text { spheroid size }\end{array}$ \\
\hline Rotating wall vessels & Create a microgravity environment & Shear force & Suitable for large scale spheroid & \\
\hline Spinner flasks & Generate dynamic fluid shear force & Shear force & & \\
\hline
\end{tabular}


and difficulty in spheroid size control. Therefore, various spheroid formation techniques using functional biomaterials have been developed (Fig. 3). For example, porous scaffolds can induce in situ spheroid formation in the pores and subsequently be co-transplanted to target tissues. The scaffolds protect spheroids not only from shear stress generated during injection, but also from oxidative stress at transplanted sites. On the other hand, nano-/microparticles (or fibers) incorporated in spheroids are beneficial to deliver drugs to cells in the core region of spheroids and create gaps improving oxygen and nutrient transportation. Indeed, advanced biomaterials improve survival and engraftment of cell spheroids and increase the potential of cell spheroids in regenerative therapy by strategizing target tissue specific engineering. Table 2 summarizes the current spheroid-based approaches according to target tissues.

\section{BONE DEFECT REGENERATION}

Bone is a mineralized connective tissue that constitutes body structure and enable mobility (25). Bone tissues are capable of sufficient self-healing for small sized damages or defects that are not over the critical size threshold ( $>2 \mathrm{~cm}$ ) (2). However, large defects, fractures, and degenerative or congenital diseases will not achieve complete healing if unaided $(26,27)$. Although autografts and allografts are gold standard treatments for bone repair, they have inevitable side effects such as morbidity, infection, and hemorrhage at the donor site (28). To overcome these limitations, engineered bone spheroids composed of stem cells (e.g., bone marrow-derived stem cells and adiposederived stem cells) have been used as micro-bone tissues and explored extensively due to their great therapeutic potential for the repair of nonhealing bone defects $(2,26,29)$. In comparison with dissociated MSCs, MSC spheroids can enhance survival in a harsh microenvironment and maintain their osteogenic potential (30). In addition, pre-osteogenic induction of MSC spheroids before transplantation can enhance the potential

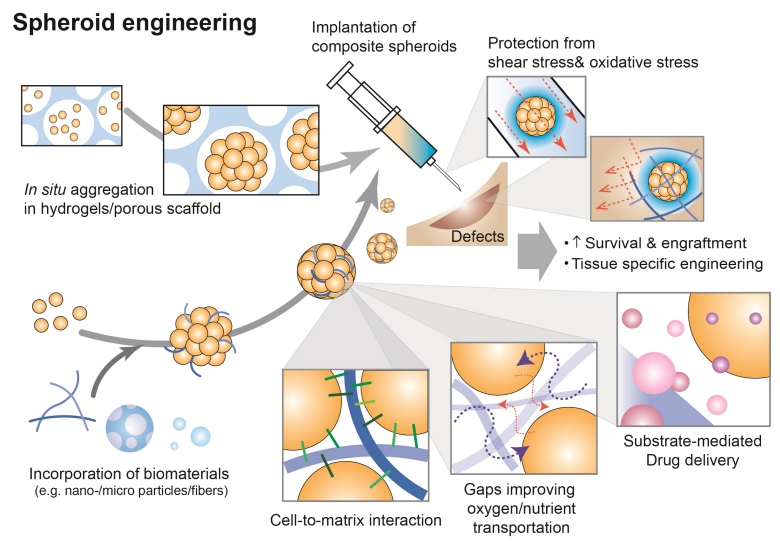

Fig. 3. Principles of biomaterials-based spheroid engineering. contribution of transplanted cells toward bone formation (31). Furthermore, biomaterials-assisted cell delivery therapies can significantly improve the regenerative capacity of stem cells by recapitulating the complex bone microenvironment.

\section{Engineering cell spheroids using 3D scaffolds}

For bone repair, various cell spheroids in combination with engineered 3D scaffolds such as hydrogels, microparticles, and electrospun fibers have been attempted $(32,33)$. Entrapment of MSC spheroids within engineered 3D scaffolds can localize cells at the implantation site, regulate cell migration from spheroids to surrounding tissues, and modulate spheroid function. The function of MSCs can be influenced by many properties of engineered 3D scaffolds including bulk mechanical properties, degradation profiles, and densities of adhesive ligands $(34,35)$. For example, Ho et al. (31) have reported that Arg-Gly-Asp (RGD) ligand density in alginate hydrogels can regulate cell adhesion and migration kinetics of osteoinduced MSC spheroids and subsequently affect their therapeutic potential. Upon ectopic implantation, MSC spheroids in higher RGD density gels can significantly facilitate bone formation than those in lower RGD density gels, suggesting that regulations of MSCs migration from spheroids are correlated with the osteogenic potential of spheroids. In another study, Whitehead et al. (36) have demonstrated that dynamic mechanical properties of viscoelastic alginate hydrogel can promote the osteogenic potential of entrapped MSC spheroids. RGD-modified alginate is either ionically or covalently crosslinked for preparing viscoelastic or elastic hydrogels, respectively. Calvaria bone defects treated with MSC spheroids entrapped in viscoelastic hydrogels exhibit significantly improved bone formation as compared to elastic hydrogel groups. Interestingly, regardless of bone morphogenetic protein-2 (BMP-2) treatment (via nanoparticles or bolus delivery), significant bone formation is shown in defects treated with spheroids in viscoelastic alginate hydrogels, suggesting that stress-relaxation of the hydrogel, rather than the internal BMP-2 signal, affects more on the osteogenic potential of entrapped MSC spheroids (36).

Due to compact cell-cell interactions of spheroids, penetration of soluble osteogenic/angiogenic cues (e.g., BMP-2, adenosine, and platelet-derived growth factor [PDGF]) into spheroids is insufficient to stimulate inner cells of spheroids. For example, spheroids cultured in media containing soluble BMP-2 exhibit differentiation only at the periphery of spheroids. On the other hand, MSC spheroids incorporating BMP-2-loaded hydroxyapatite (HA) nanoparticles exhibit greater alkaline phosphatase activity and more uniform spatial expression of osteocalcin than spheroids with uncoated HA nanoparticles (37). Similarly, incorporation of adenosine-modified microfibers to MSC spheroids can stimulate adenosine $2 \mathrm{~b}$ receptor signaling of MSCs thus significantly upregulating osteogenic markers (i.e., Runt-related transcription factor 2 (RUNX2), Osteopontin (OPN), Osteocalcin $(O C N)$, and Osterix $(O S X)$ ) with improved mineral deposition in vitro. In comparison with unmodified microfibers/spheroid 
Table 2. Summary of biomaterials and strategies of spheroid engineering according to the target applications

\begin{tabular}{|c|c|c|c|c|}
\hline Target & Cell type & $\begin{array}{l}\text { Functional } \\
\text { biomaterials }\end{array}$ & $\begin{array}{l}\text { A method for } \\
\text { spheroid formation }\end{array}$ & Strategy \\
\hline \multirow[t]{6}{*}{$\begin{array}{l}\text { Bone defect } \\
\text { repair }\end{array}$} & hBMSC & RGD-modified alginate gels (31) & Microwells & $\begin{array}{l}\text { Controlling MSC migration from spheroids to } \\
\text { enhance spheroid osteogenic potential }\end{array}$ \\
\hline & hBMSC & Alginate hydrogel (36) & Microwells & $\begin{array}{l}\text { Applying dynamic mechanical stimulation to } \\
\text { spheroids for enhancing osteogenic potential } \\
\text { of MSC }\end{array}$ \\
\hline & hADSC & $\begin{array}{l}\text { Adenosine and polydopamine } \\
\text { coated PLLA fragmented fibers } \\
\text { (38) }\end{array}$ & Centrifugation & $\begin{array}{l}\text { Scaffolds-mediated adenosine delivery to } \\
\text { improve osteogenic differentiation of MSCs }\end{array}$ \\
\hline & rbBMSC & Silk fibroin microfiber (39) & Centrifugation & $\begin{array}{l}\text { Creating gaps in spheroids, leading to enhanced } \\
\text { transportation of oxygen and nutrients to the } \\
\text { core region }\end{array}$ \\
\hline & hADSC & $\begin{array}{l}\text { Biomineral-coated PLLA } \\
\text { fragmented fibers }(41)\end{array}$ & Centrifugation & $\begin{array}{l}\text { Accelerating osteogenic differentiation by } \\
\text { providing bone-like mineralized environments }\end{array}$ \\
\hline & hADSC & $\begin{array}{l}\text { PDGF/biomineral-coated PLLA } \\
\text { fragmented fibers }(42)\end{array}$ & Centrifugation & $\begin{array}{l}\text { Providing bone-mimicking multiple factors for } \\
\text { vascularized bone regeneration }\end{array}$ \\
\hline \multirow[t]{4}{*}{$\begin{array}{l}\text { Cartilage defect } \\
\text { repair }\end{array}$} & rBMSC & Magnetic nanoparticles (54) & $\begin{array}{l}\text { Magnetic condensation } \\
\text { using magnetic devices }\end{array}$ & $\begin{array}{l}\text { Controlling sizes and patterns of spheroids at } \\
\text { the millimetric scale by using magnetic } \\
\text { devices }\end{array}$ \\
\hline & rbADSC & $\begin{array}{l}\text { PLGA/chitosan porous scaffold } \\
\text { (55) }\end{array}$ & In situ aggregation in pores & $\begin{array}{l}\text { Forming denser mass of spheroids in the } \\
\text { scaffold, leading to enhanced chondrogenic } \\
\text { differentiation capacity of stem cells }\end{array}$ \\
\hline & hADSC & $\begin{array}{l}\text { TGF- } \beta 3 \text { and FN adsorbed } \\
\text { graphene oxide sheet (58) }\end{array}$ & Hanging-drop & $\begin{array}{l}\text { Providing a cell-adhesion substrate and } \\
\text { simultaneously delivering chondrogenic }\end{array}$ \\
\hline & UCB-MSC & $\begin{array}{l}\text { hFDM and TGF- } \beta 1 \text {-coated } \\
\text { PLGA/PLLA microfiber (59) }\end{array}$ & Non-adherent plates & $\begin{array}{l}\text { growth factors for improving chondrogenic } \\
\text { differentiation of stem cells }\end{array}$ \\
\hline \multirow[t]{3}{*}{$\begin{array}{l}\text { Critical limb } \\
\text { ischemia repair }\end{array}$} & UCB-MSC & $\begin{array}{l}\text { Hyaluronic acid/alginate } \\
\text { core-shell microcapsules (64) }\end{array}$ & Microencapsulation & $\begin{array}{l}\text { Encapsulating spheroids to protect and retain } \\
\text { the cells from harsh environments after } \\
\text { transplantation }\end{array}$ \\
\hline & hADSC & $\begin{array}{l}\text { Poly(L-glutamic acid)/PEG-based } \\
\text { porous hydrogel }(65)\end{array}$ & In situ aggregation in pores & $\begin{array}{l}\text { In situ spheroid formation via gel-sol transition } \\
\text { in vivo, protecting spheroids from shear stress } \\
\text { during injection }\end{array}$ \\
\hline & RAW 264.7 & $\begin{array}{l}\text { Chrysin-encapsulated fiber } \\
\text { fragments (68) }\end{array}$ & $\begin{array}{l}\text { Electrosprayed } \\
\text { microcapsulation }\end{array}$ & $\begin{array}{l}\text { Promoting vascular anastomosis via } \\
\text { chronological shifting from } \mathrm{M} 1 \text { to } \mathrm{M} 2 \\
\text { phenotypes, regulated by chrysin delivery }\end{array}$ \\
\hline \multirow[t]{3}{*}{ Cardiac repair } & hiPSC-CM & Silicon nanowires $(70,71)$ & Microwells & $\begin{array}{l}\text { Incorporating electrically conductive } \\
\text { biomaterials to achieve synchronized and } \\
\text { enhanced contraction of cardiac spheroids }\end{array}$ \\
\hline & & & & $\begin{array}{l}\text { Used both exogenous and endogenous } \\
\text { electrical stimuli for advanced structural and } \\
\text { functional development of cardiac spheroids }\end{array}$ \\
\hline & hBMSC & $\begin{array}{l}\text { Reduced graphene oxide flake } \\
\text { (72) }\end{array}$ & Hanging-drop & $\begin{array}{l}\text { Incorporating electroconductive biomaterials to } \\
\text { spheroids for enhancing paracrine factors and } \\
\text { connexin } 43 \text { expression }\end{array}$ \\
\hline \multirow[t]{5}{*}{$\begin{array}{l}\text { Islet } \\
\text { transplantation }\end{array}$} & $\begin{array}{l}\text { Human } \\
\text { pancreatic islets }\end{array}$ & $\begin{array}{l}\text { ECM hydrogels made of porcine } \\
\text { decellularized tissues (83) }\end{array}$ & Encapsulation & $\begin{array}{l}\text { Recapitulating the in vivo peri-islet niche to } \\
\text { enhance cell survival and functions }\end{array}$ \\
\hline & $\begin{array}{l}\text { Mouse pancreatic } \\
\text { islets }\end{array}$ & $\begin{array}{l}\text { Chondroitin sulfate incorporated } \\
\text { starPEG (80) }\end{array}$ & Nanocoating & $\begin{array}{l}\text { Nanocoating of islets to reduce blood } \\
\text { coagulation, improve islet cells survival, and } \\
\text { protect against disruption }\end{array}$ \\
\hline & & $\begin{array}{l}\text { Fas ligand-conjugated PEG } \\
\text { microgel (81) }\end{array}$ & Microencapsulation & \multirow{2}{*}{$\begin{array}{l}\text { Local immunomodulation to avoid acute } \\
\text { rejection of islet allografts, avoiding the need } \\
\text { for systemic chronic immunosuppression }\end{array}$} \\
\hline & & $\begin{array}{l}\text { Programmed cell } \\
\text { death-1-conjugated PEG } \\
\text { microgel (79) }\end{array}$ & Microencapsulation & \\
\hline & & $\begin{array}{l}\text { TGF- } \beta 1 \text {-loaded PLG } \\
\text { microporous scaffold (82) }\end{array}$ & In situ aggregation in pores & $\begin{array}{l}\text { Localized TGF- } \beta 1 \text { delivery to modulate the } \\
\text { immunological environment of transplanted } \\
\text { sites }\end{array}$ \\
\hline
\end{tabular}


Table 2. Continued

\begin{tabular}{clccc}
\hline Target & Cell type & $\begin{array}{c}\text { Functional } \\
\text { biomaterials }\end{array}$ & $\begin{array}{c}\text { A method for } \\
\text { spheroid formation }\end{array}$ & Strategy \\
\hline $\begin{array}{l}\text { Hair follicle } \\
\text { regeneration }\end{array}$ & hDPC & Polyvinyl alcohol (PVA) (91) & PVA-coated plates & $\begin{array}{c}\text { Developed a controllable spheroid formation } \\
\text { technique }\end{array}$ \\
mDPC & $\begin{array}{c}\text { Chitosan/PVA nanofiber } \\
\text { sponge (92) } \\
\text { Gelatin and alginate (93) }\end{array}$ & In situ aggregation in pores & $\begin{array}{c}\text { Leveloped a technique for controllable and } \\
\text { scalable spheroids formation } \\
\text { Developed a tunable and scalable spheroid } \\
\text { formation model by inducing aggregation of } \\
\text { nanoencapsulated cells }\end{array}$ \\
\hline
\end{tabular}

MSC, mesenchymal stem cell; hBMSC, human bone marrow-derived MSC; hADSC, human adipose-derived stem cell; rbBMSC, rabbit bone marrow-derived MSC; rbADSC, rabbit adipose-derived stem cell; UCB-MSC, human umbilical cord blood-derived MSC; hiPSC-CM, human induced pluripotent stem cell-derived cardiomyocytes; hDPC, human dermal papilla cells; mDPC, mouse DPC; RGD, Arg-Gly-Asp; PLLA, poly (L-lactic acid); PDGF, platelet-derived growth factor; PLGA, poly(lactic-co-glycolic acid); TGF, transforming growth factor; FN, fibronectin; hFDM, human lung fibroblast decellularized ECM; PEG, polyethylene glycol; PLG, poly(lactide-co-glycolide).

composite, in vivo calvarial bone formation of adenosinemodified microfibers/spheroid composite is greater with thick and compact bone tissues similar to that of native bone tissues (38).

3D scaffolds (e.g., microparticles and microfibers) have been incorporated within cell alone spheroids not only to deliver bioactive molecules, but also to improve viability of inner cells in spheroids $(39,40)$. In general, inadequate mass transport and development of a hypoxic core within spheroids limit their long-term culture and practical application. Incorporation of microparticles or microfibers generates relatively less compact spheroids of cells, thus improving cell viability. Microfibers are especially beneficial for bone tissue engineering due to their hierarchically organized architecture mimicking bone microenvironment. Ahmad et al. (41) have fabricated mineralized poly (L-lactic acid) (PLLA) microfibers architecturally and chemically mimicking bone microenvironment. These mineralized microfibers can control the differentiation of stem cells by regulating $\mathrm{Ca}^{2+}$ influx and $\mathrm{PO}_{4}{ }^{3-}$ involved $\mathrm{A} 2 \mathrm{~b}$ adenosine receptor activation during osteogenic differentiation of MSCs. Thus, even without osteogenic supplements in the media, MSC spheroids with mineralized microfibers exhibited significantly enhanced expression of osteogenic genes including Runx2, OPN, and OCN as compared to the no mineralized microfiber group. Furthermore, mineralized microfibers incorporating PDGF can enhance both vascularization and bone regeneration capacity of MSC spheroids. At two months after transplantation of MSC spheroids with mineralized microfibers in a mouse calvarial defect model, the regenerated bone area was significantly increased and many capillaries and arterioles were observed in the presence of PDGF incorporated on microfibers (42).

\section{CARTILAGE DEFECT REGENERATION}

Cartilage healing is extremely slow due to its avascular properties (43). Once cartilage is damaged, it is often healed with fibrocartilage scar tissue which remains a challenge (44). Hyaline cartilage consists of specialized ECM including collagen type II (COL II) and proteoglycans, while fibrocartilage produces more collagen type I (COL I) but less COL II and exhibits inferior capacity to support high dynamic compressive loads (45-47). Thus, it is important to engineer cell spheroids to produce hyaline cartilage-specific ECM for successful cartilage repair.

\section{Condensation}

In vitro spheroids or aggregates formation of MSCs can trigger the commitment for the chondrogenic lineage by mimicking in vivo "condensation" step, a critical event that occurs during limb development $(48,49)$. Conventional cell condensation techniques include centrifugation (50), micromass culture (51), hanging drop method (52), and continuous agitation of a suspension culture (53). Recently, magnetic condensation technique has drawn much attention due to its ease of shaping multicellular organization according to the size and shape of cartilage defects (54). Fayol et al. (54) have reported that magnetically driven MSC chondrogenesis can produce more cartilage matrix including COL II and aggrecan than pellets prepared by centrifugation. Further magnetic fusion of MSC spheroids can yield millimetric scale of large tissues suitable for repairing large cartilage defects.

Similarly, in situ formation of MSC spheroids inside a 3D porous scaffold is another strategy for engineering cartilage tissue substitute. Cell repulsive scaffolds such as chitosan film can generate spontaneous cellular aggregation due to their low adhesive force between cells and scaffolds (55). The highly hydrophilic layer of scaffolds can tightly bind to water via hydrogen bonds or electrostatic interaction, thus achieving a non-fouling ability. For example, a porous scaffold made of zwitterionic poly (L-glutamic acid)-chitosan co-polymer (PLGA-CS) can generate MSC spheroids with diameter of $80-100 \mu \mathrm{m}$ in situ (55). MSC spheroids in PLGA-CS scaffold exhibited significantly upregulated chondrogenic genes (COL II and glycosaminoglycans (GAG)) expression and decreased expression of a marker for fibrocartilage (COL $I$ ) as compared to MSCs attached and spread on scaffolds. Furthermore, at 12 weeks post-implan- 
tation, neo-cartilage at defects treated with the PLGA-CS scaffold carrying MSC spheroids exhibited similar level of GAGs and COL II and low level of COL I as a normal cartilage. These results suggest that in situ spheroid formation in porous scaffolds not only can promote chondrogenesis of MSCs, but also can facilitate hyaline-like cartilage regeneration with reduced fibrous tissue formation.

\section{Substrate-mediated TGF- $\beta$ delivery}

Transforming growth factor beta (TGF- $\beta$ ) superfamily plays essential roles in all phases of chondrogenesis, mesenchymal condensation, chondrocyte proliferation, extracellular matrix deposition, and finally terminal differentiation (56). TGF- $\beta$ is a key initiator of chondrogenesis. Cellular condensation is strongly stimulated by TGF- $\beta$-induced elevation of $\mathrm{N}$-cadherin expression (49). In addition, TGF- $\beta$ can stimulate the proliferation of chondroblasts and deposition of cartilage-specific ECMs (e.g., aggrecan and collagen type II). It has been demonstrated that exogenous TGF- $\beta$ treatment could enhance chondrogenic differentiation of MSCs (57). However, as MSCs form spheroids, it is difficult to achieve efficient TGF- $\beta$ delivery to cells inside spheroids due to diffusional limitation of TGF- $\beta$. To overcome this limitation, substrate-mediated TGF- $\beta$ delivery systems have been developed. For example, Yoon et al. (58) have loaded TGF- $\beta 3$ to fibronectin (FN)-coated graphene oxide (GO) sheets (0.5-1 $\mu \mathrm{m}$ size). FN and TGF- $\beta 3$ can be adsorbed onto GO sheets via hydrophobic interactions and electrostatic interactions. As FN-/TGF- $\beta 3$-adsorbed GO sheets are incorporated into MSC spheroids, they not only provide cell-ECM interaction, but also overcome the diffusional limitation of TGF- $\beta 3$, resulted in significantly enhanced chondrogenic differentiation of MSCs in vitro. Similarly, Kim et al. (59) have developed a TGF- $\beta 1$ delivery system based on PLGA/PLLA microfiber scaffolds. The surface of the microfiber scaffold was modified with human fibroblast-derived decellularized ECM (hFDM) to mimic in vivo cell-matrix interactions. Heparin was subsequently grafted onto the hFDM scaffold for achieving high loading amount of TGF- $\beta 1$. Such TGF- $\beta 1$-loaded hFDM scaffold exhibited a continuous release of TGF- $\beta 1$ for up to 28 days. Furthermore, at 12 weeks post-implantation, MSC spheroids with TGF $\beta 1$-loaded hFDM scaffolds significantly increased cartilage regeneration of defects in a rabbit knee articular cartilage defect model.

\section{CRITICAL LIMB ISCHEMIA REPAIR}

Cell-based therapies are promising for reconstructing blood vessels and restoring blood perfusion of various injuries or diseases. MSCs have been extensively used for critical limb ischemia (CLI) repair due to their proangiogenic and immunoregulatory functions $(60,61)$. However, low retention and poor viability of transplanted cells at the implantation site limit their therapeutic effects. It has been known that MSC spheroids exhibit higher cell viability in vivo than single cell transplantation. More importantly, MSC spheroids produce more angiogenic growth factors and cytokines (e.g., angiopoietin, angiopoietin-2, FGF2, HGF, and VEGF) by activating hypoxiainduced paracrine secretion signalling $(4,62,63)$. Furthermore, it has been demonstrated that in situ MSC spheroids encapsulation in biomaterials can physically protect spheroids during and after transplantation $(64,65)$. For example, Park et al. (64) have reported that when MSCs are encapsulated in hyaluronic acid (HA)/alginate (Alg) core-shell microcapsules, cells can spontaneously form spheroids in the $\mathrm{HA}$ core region, while the outer alginate shell provides a protection layer. MSC spheroids encapsulated in the microcapsule can significantly promote in vivo angiogenesis as compared to MSC alone spheroids. This is attributed to the greater retention of MSC spheroids encapsulated in the microcapsule. In another study, Hong et al. (65) have developed an all-in-one platform that supports in situ spheroid formation and injection at once. The platform uses poly (L-glutamic acid)-based hydrogels crosslinked with disulfide bonds. Cells can spontaneously form spheroids in pores of the hydrogel due to non-fouling features of the hydrogel. For in vivo implantation, hydrogels could be turned into solution by cleaving the disulfide bonding via a reductant (e.g., glutathione) and co-injected with spheroids. This platform not only supports spheroid formation, but also improves survival and engraftment by protecting spheroids during and after the transplantation.

Recently, macrophages have been emerged as a promising cell source for CLI repair. Macrophages are primary effector cells of the immune system that dominantly provide cytokines to regulate angiogenesis and matrix remodelling during tissue repair $(66,67)$. Ran et al. (68) have generated macrophage spheroids with chrysin-loaded fibers. These fibers not only can serve as a scaffold, but also can release chrysin for regulating the phenotypic transformation of macrophages from proinflammatory $\mathrm{M} 1$ to anti-inflammatory $\mathrm{M} 2$. In vivo results have revealed that M2 macrophage polarization of spheroids can induce a full recovery of hind-limb ischemia to normal limbs. It has been also proven that macrophage spheroids transplantation can lead to greater limb salvage than single macrophage administration (68).

\section{CARDIAC REPAIR}

Cardiac muscle allows the heart to pump blood through circulatory system. Its dysfunction can lead to heart failure. Myocardial infarction (Ml) can cause loss of gap junctionexpression cardiac cells and cardiac fibrosis, thus increasing the risk of arrhythmia (69). For functional recovery of infarcted hearts, cardiac spheroids composed of MSCs or cardiomyocytes have been implanted into the ischemic myocardium (70-72). Although cardiac spheroids can restore cardiac functions of infarcted hearts, they often yield unsynchronized contraction, leading to a potential risk of arrhythmia. To overcome this limitation, electrically conductive materials can be incorporated into cardiac spheroids. For example, incorporating silicon nanowires into cardiac spheroids can improve the formation of 
an electrically conductive network in spheroids, leading to significantly synchronized and enhanced contraction as compared to non-incorporated cardiac spheroids (Fig. 4) (71). Furthermore, in combination with external electrical stimulation (recapitulating natural pacemaker-initiated excitation of cardiomyocyte contraction), silicon nanowire-incorporated cardiac spheroids can further improve cell-cell junction formation and the development of a contractile machinery and decrease the spontaneous beat rate of spheroids, thus reducing arrhythmogenic potential (70). Park et al. (72) have reported that implantation of reduced graphene oxide (RGO) flakes-incorporated MSC spheroids into infarcted hearts can enhance cardiac repair as compared to implantation of RGO flakes or MSC spheroids alone. The incorporation of RGO flakes into spheroids not only can provide electrically conductive cues, but also can induce tighter cell-cell compaction and more reparative paracrine factor secretion than non-incorporated spheroids.

\section{PANCREAS REGENERATION}

Type 1 diabetes is a chronic autoimmune disease. Due to T-cell mediated attack, $\beta$ cells in pancreatic islets cannot produce adequate insulin, resulting in hyperglycemia (73). Although insulin uptake through intensive insulin injection or pumps can effectively decrease blood glucose levels, exogenous insulin therapy has a risk of complications such as hypoglycemia and ketoacidosis $(73,74)$. In addition, exogenous insulin uptake cannot ameliorate symptoms of patients with type 1 diabetes who exhibit severe hypoglycemia complicated by impaired hypoglycemia awareness or excessive glycemic lability (74). Although pancreas transplantation is the best option, it is an invasive surgery that needs lifelong immunosuppression to relieve massive immune attack to the graft. More importantly, the scarcity of donor pancreas limits its extensive application. Transplantation of allogenic islet or stem cell-derived islets is a promising alternative that can avoid an invasive surgery by directly injecting islets to the hepatic portal vein (75). However, islet anoikis can be triggered during an ex vivo isolation. In addition, in vivo immune rejection still remains a challenge (76). Recently, various biomaterials-based approaches for islet transplantation have been explored to enhance islet survival and functions and decrease host immune rejection of allogenic islet transplants (77-83).

In the pancreas, islets of Langerhans are surrounded by a layer of ECM defined as the peri-insular basement membrane composed of collagen type IV, laminin, and fibronectin (84).
A
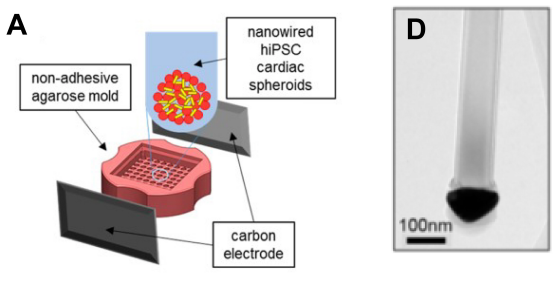

B

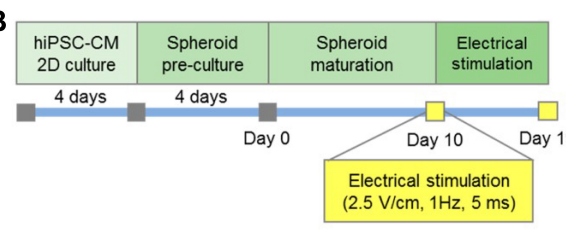

C

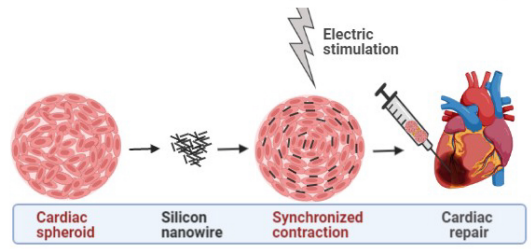

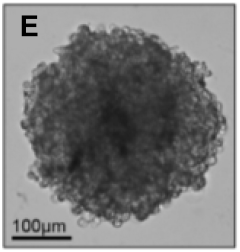
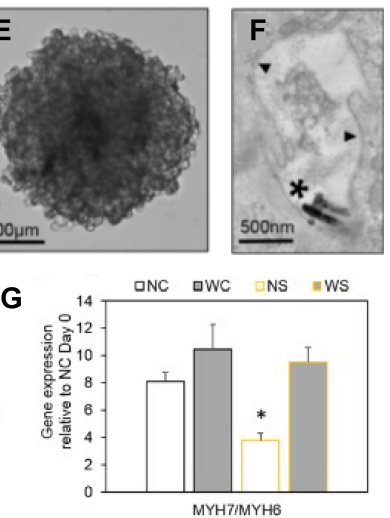

H

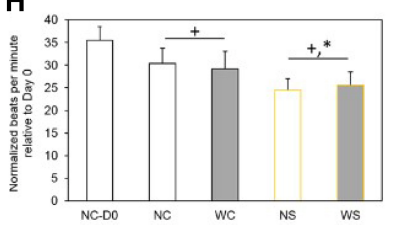

Fig. 4. Cardiac spheroids formation with silicon nanowires and maturation with external electrical stimulation. (A) Schematic illustrations of a setup for external electrical stimulation to cardiac spheroids. (B) Timeline of in vitro conditioning. (C) Silicon nanowire incorporation to spheroids could improve maturation with electrical stimulation to be beneficial for cardiac repair. (D-F) Transmission electron micrograph images showing (D) structures of n-type doped silicon nanowires (scale bar, $100 \mathrm{~nm}$ ), (E) composite spheroids formed with human induced pluripotent stem cells (hiPSCs) and silicon nanowires (scale bar, $100 \mu \mathrm{m}$ ), and (F) silicon nanowires located in extracellular space (scale bar, $500 \mathrm{~nm}$ ). (G) Expression of genes related to contractile machinery in hiPSC cardiac spheroids (beta myosin heavy chain, MYH7; alpha myosin heavy chain, MYH6). NC, unwired spheroid; WC, wired spheroid; NS, unwired spheroid with stimulation; WS, wired spheroid with stimulation. $(\mathrm{H})$ Beat rate of hiPSC cardiac spheroids, showing that electrical stimulation significantly reduced spontaneous beat rate in spheroids incorporating silicon nanowires and electrically stimulated (WS). Taken together, tissue level functional development would be beneficial for reduced pacemaking and arrhythmic risk after transplantation. Adapted with permission from Richards et al. (70). Copyright (2016) American Chemical Society. 
During an enzymatic isolation of islets from pancreas tissues, the interaction between the islet and the ECM is disrupted. Subsequent reduction of cell adhesion can trigger $\beta$ cell apoptosis and decrease insulin secretion (77). To overcome this limitation, pancreatic decellularized ECM (dECM) hydrogels have been used because they can mimic physicochemical cues of in vivo peri-niche (85). For example, Jiang et al. (83) have reported that porcine pancreas $\mathrm{dECM}$ hydrogel exhibits similar viscoelastic properties to original pancreas tissues. More importantly, encapsulation of human or rat islets within porcine pancreas-derived $\mathrm{dECM}$ hydrogels can improve the functional stability of islets as compared to the standard culture condition (freely suspended in non-adherent dishes).

Encapsulation islets in biomaterials can protect islets from a harsh microenvironment (e.g., immune attack, inflammatory conditions, etc.). Alginate microencapsulation of islets has been extensively used due to its ease of use. Alginate can be rapidly crosslinked in the presence of divalent cations (e.g. $\mathrm{Ca}^{2+}$ ) (86). However, the size of alginate microspheres $(0.5-1.5 \mathrm{~mm})$ often exceeds the physiological oxygen diffusion range, resulting in reduced islet survival and function (78). Yang et al. (80) have reported that a nanocoating of islets with chondroitin sulfate (CS)-incorporated starPEG (CS-PEG) can protect islets under pro-inflammatory conditions. CS-PEG can covalently conjugate to amine groups of the islet cell membrane via pseudoorthogonal chemistry, resulting in a CS-PEG layer with thickness of $50 \mathrm{~nm}$ on the islet $(\sim 100 \mu \mathrm{m})$. The nanocoating makes a minimal alteration of the islet volume and allows excellent oxygen diffusion. More importantly, such CS-PEG coating can significantly reduce blood coagulation and inflammationrelated islet cell death and potentially alleviate instant bloodmediated inflammatory reaction during an islet transplantation.

Islet transplantation is limited not only by host immune responses, but also by toxic effects of chronic immunosuppression required to control immune rejection (87). Recently, biomaterials-assisted strategies have focused on providing a local immunosuppression microenvironment. Biomaterials presenting checkpoint proteins (e.g., programmed cell death-1 ligand [PD-L1] and Fas ligand [FasL]) can modulate local immune responses and avoid the need for systemic chronic immunosuppression. For example, Headen et al. (81) have demonstrated that co-transplantation of FasL-presenting microgels and allogenic islets in the epididymal fat pad of diabetic mice can result in graft tolerance and function without chronic immunosuppression. Free FasL can be rapidly cleared from the transplant site $(60 \%$ reduction at day 1 post-implantation) while FasL-presenting microgels can last much longer at the implantation site over 11 days. Similarly, Coronel et al. (79) have reported that PD-L1-presenting microgels can improve local retention of PD-L1 over 3 weeks in vivo. Therefore, PD-L1presenting microgels can achieve local induction of allograft islet acceptance in a murine diabetic model. Liu et al. (82) have reported that TGF- $\beta 1$-releasing scaffolds can significantly decrease inflammatory cytokines (i.e., TGF- $\alpha$, IL-2, and MCP-1) and subsequent leukocyte infiltration to the implantation site, resulting in a delayed immune rejection of allogenic islets.

\section{HAIR FOLLICLE REGENERATION}

Hair loss is a significant concern of human disorder regardless of age and gender. This can be attributed to aging, environmental reasons, stress, or the lack of hair follicle recovery due to poor tissue regeneration of injured tissues (88). Hair follicle is an ectodermal organ composed of two main parts, including the epithelium of keratinocytes and the mesenchyme of dermal papilla (DP) cells (89). DP cells are known as a master regulator of HF cycle. They give instructive signals to epithelial bulge to initiate follicle formation, growth, and proliferation during HF regeneration (90). Currently, DP spheroids implantation is the best option for HF regeneration. To construct efficient and functional DP spheroids, non-adhesive scaffolds made of hydrophilic polymers have been used. After cells are seeded onto non-adhesive/non-fouling scaffolds, they rather spontaneously aggregate to each other and form a spheroid (91). Huang et al. (91) have developed a scalable production platform for DP spheroid generation based on a hydrophilic polymer, polyvinyl alcohol (PVA), and demonstrated effects of DP spheroid size on hair follicle regeneration. DP cells in hanging drops (a conventional method) can form multiple small aggregates. On the other hand, DP cells seeded onto PVA-coated tubes can spontaneously aggregate into one single spheroid due to their poor cell adhesion to hydrophilic PVA. An in vivo study has revealed that both human and rat DP spheroids could induce hair follicle neogenesis and larger DP spheroids exhibit higher hair follicle inductivity in dorsal hypodermis of mice (91). Similarly, Zhang et al. (92) have used a chitosan/PVA nanofibrous sponge with large open pores for generating DP spheroids. The sponge possesses interconnected pores $(21-25 \mu \mathrm{m})$ that could capture cells, resulting in formation of spheroids in pores. DP spheroids implanted into BALB/C nude mice can induce hair follicle neogenesis at 4 weeks after implantation. Larger sizes of DP spheroids have regenerated more hair shafts (92).

Wang et al. (93) have also developed a tunable and scalable DP spheroid formation platform by aggregating nanoencapsulated single cells. DP cells were coated with type A gelatin (positively charged) and alginate (negatively charged) by layer-by-layer assembly. Gelatin/alginate-coated DP cells were ionically crosslinked in the presence of divalent cations (i.e., $\mathrm{Ca}^{2+}$ ), generating DP spheroids in a scalable manner. More importantly, when cells were coated with gelatin/alginate, even high-passaged (P8) DP cell spheroids could maintain their ability of hair follicle induction in vivo while P8 DP cells without the coating could not regenerate hair follicles at all.

\section{FUTURE DIRECTIONS}

A spheroid formation is indeed a promising approach to mimic in vivo microenvironments. Moreover, biomaterials-assisted 
spheroid engineering can improve clinical therapeutic effects of spheroids even further. Biomaterials can provide both interior and exterior cues to spheroids for improving cellular functions and survival. Nano- and micro-particles incorporated to spheroids can deliver bioactive molecules to cells in spheroids. Hydrogels and fibers can facilitate spontaneous spheroid formation and protect spheroids from harsh environment of transplanted sites. Additionally, functional moieties (e.g., cell adhesion ligands and immunomodulatory proteins) are often decorated to those biomaterials for further regulation of host cell migration and immune responses during the healing process. So far, many studies have focused on recapitulating chemical or/and structural features of in vivo microenvironments. Future studies should consider tailoring physical features of scaffolds (e.g., stiffness, topography, mechanical stability, and degradation profiles) that compensate dynamic mechanical alterations of injured tissues. There is emerging evidence that physical cues applied to injured tissues are critical to regulate tissue repair and even immune responses via mechanotransduction (94-97). However, their roles in 3D spheroids have not been fully investigated yet. Nano- and micro-particles based platforms can generate internal forces inside spheroids and regulate cell-to-cell and cell-to-matrix interactions. Scaffolds encapsulating spheroids would primarily crosstalk with peripheral cells of spheroids and regulate cell migration and integration with host tissues. This approach could open a new frontier in regenerative therapy.

\section{ACKNOWLEDGEMENTS}

This research was supported by the research fund of Dankook University in 2020.

\section{CONFLICTS OF INTEREST}

The authors have no conflicting interests.

\section{REFERENCES}

1. Iturriaga L, Hernaez-Moya R, Erezuma I, Dolatshahi-Pirouz A and Orive G (2018) Advances in stem cell therapy for cartilage regeneration in osteoarthritis. Expert Opin Biol Ther 18, 883-896

2. Watanabe Y, Harada N, Sato K, Abe S, Yamanaka K and Matushita T (2016) Stem cell therapy: is there a future for reconstruction of large bone defects? Injury 47, S47-S51

3. Lee YB, Kim EM, Byun H et al (2018) Engineering spheroids potentiating cell-cell and cell-ECM interactions by selfassembly of stem cell microlayer. Biomaterials $165,105-120$

4. Lee JH, Han YS and Lee SH (2016) Long-duration threedimensional spheroid culture promotes angiogenic activities of adipose-derived mesenchymal stem cells. Biomol Ther (Seoul) 24, 260-267

5. Baldari S, Di Rocco G, Piccoli M, Pozzobon M, Muraca $M$ and Toietta G (2017) Challenges and strategies for improving the regenerative effects of mesenchymal stromal cell-based therapies. Int J Mol Sci 18, 2087

6. Amer $M H$, Rose FRAJ, Shakesheff $K M$, Modo $M$ and White LJ (2017) Translational considerations in injectable cell-based therapeutics for neurological applications: concepts, progress and challenges. NPJ Regen Med 2, 23

7. Sandvig I, Gadjanski I, Vlaski-Lafarge M et al (2017) Strategies to enhance implantation and survival of stem cells after their injection in ischemic neural tissue. Stem Cells Dev 26, 554-565

8. Fennema E, Rivron N, Rouwkema J, van Blitterswijk C and de Boer J (2013) Spheroid culture as a tool for creating 3D complex tissues. Trends Biotechnol 31, 108-115

9. Mahmood TA, de Jong R, Riesle J, Langer $R$ and van Blitterswijk CA (2004) Adhesion-mediated signal transduction in human articular chondrocytes: the influence of biomaterial chemistry and tenascin-C. Exp Cell Res 301, 179188

10. Bierwolf J, Lutgehetmann M, Feng K et al (2011) Primary rat hepatocyte culture on 3D nanofibrous polymer scaffolds for toxicology and pharmaceutical research. Biotechnol Bioeng 108, 141-150

11. Ryu NE, Lee SH and Park H (2019) Spheroid culture system methods and applications for mesenchymal stem cells. Cells 8, 1620

12. Barthes J, Ozcelik H, Hindie M, Ndreu-Halili A, Hasan A and Vrana NE (2014) Cell microenvironment engineering and monitoring for tissue engineering and regenerative medicine: the recent advances. Biomed Res Int 2014, 921905

13. Edmondson R, Broglie JJ, Adcock AF and Yang L (2014) Three-dimensional cell culture systems and their applications in drug discovery and cell-based biosensors. Assay Drug Dev Technol 12, 207-218

14. Kim SJ, Kim EM, Yamamoto M, Park H and Shin H (2020) Engineering multi-cellular spheroids for tissue engineering and regenerative medicine. Adv Healthc Mater, e2000608

15. Liu D, Chen S and Win Naing M (2021) A review of manufacturing capabilities of cell spheroid generation technologies and future development. Biotechnol Bioeng 118, 542-554

16. Bialkowska K, Komorowski P, Bryszewska M and Milowska K (2020) Spheroids as a type of three-dimensional cell cultures-examples of methods of preparation and the most important application. Int J Mol Sci 21, 6225

17. Kwon SH, Bhang SH, Jang HK, Rhim T and Kim BS (2015) Conditioned medium of adipose-derived stromal cell culture in three-dimensional bioreactors for enhanced wound healing. J Surg Res 194, 8-17

18. Ong CS, Zhou X, Han J et al (2018) In vivo therapeutic applications of cell spheroids. Biotechnol Adv 36, 494-505

19. Mathur A, Ma Z, Loskill P, Jeeawoody S and Healy KE (2016) In vitro cardiac tissue models: current status and future prospects. Adv Drug Deliv Rev 96, 203-213

20. Zhu L, Luo D and Liu Y (2020) Effect of the nano/ microscale structure of biomaterial scaffolds on bone regeneration. Int J Oral Sci 12, 6

21. Ko JY, Park JW, Kim J and Im GI (2021) Characterization of adipose-derived stromal/stem cell spheroids versus single-cell suspension in cell survival and arrest of osteoarthritis progression. J Biomed Mater Res A 109, 869-878 
22. Yang C, Tibbitt MW, Basta L and Anseth KS (2014) Mechanical memory and dosing influence stem cell fate. Nat Mater 13, 645-652

23. Duval K, Grover H, Han LH et al (2017) Modeling physiological events in 2D vs. 3D cell culture. Physiology (Bethesda) 32, 266-277

24. Jensen $C$ and Teng $Y$ (2020) Is it time to start transitioning from 2D to 3D cell culture? Front Mol Biosci 7, 33

25. Florencio-Silva R, Sasso GR, Sasso-Cerri E, Simoes MJ and Cerri PS (2015) Biology of bone tissue: structure, function, and factors that influence bone cells. Biomed Res Int 2015,421746

26. Perez JR, Kouroupis D, Li DJ, Best TM, Kaplan L and Correa D (2018) Tissue engineering and cell-based therapies for fractures and bone defects. Front Bioeng Biotechnol 6, 105

27. Winkler T, Sass FA, Duda GN and Schmidt-Bleek K (2018) A review of biomaterials in bone defect healing, remaining shortcomings and future opportunities for bone tissue engineering: The unsolved challenge. Bone Joint Res 7, 232-243

28. Younger EM and Chapman MW (1989) Morbidity at bone graft donor sites. J Orthop Trauma 3, 192-195

29. Oryan A, Kamali A, Moshiri A and Baghaban Eslaminejad M (2017) Role of mesenchymal stem cells in bone regenerative medicine: what is the evidence? Cells Tissues Organs 204, 59-83

30. Murphy KC, Fang SY and Leach JK (2014) Human mesenchymal stem cell spheroids in fibrin hydrogels exhibit improved cell survival and potential for bone healing. Cell Tissue Res 357, 91-99

31. Ho SS, Keown AT, Addison B and Leach JK (2017) Cell migration and bone formation from mesenchymal stem cell spheroids in alginate hydrogels are regulated by adhesive ligand density. Biomacromolecules 18, 4331-4340

32. Chahal S, Kumar A and Hussian FSJ (2019) Development of biomimetic electrospun polymeric biomaterials for bone tissue engineering. A review. J Biomater Sci Polym Ed 30, 1308-1355

33. Mao Y, Zhao Y, Guan J et al (2020) Electrospun fibers: an innovative delivery method for the treatment of bone diseases. Expert Opin Drug Deliv 17, 993-1005

34. Bhattarai DP, Aguilar LE, Park CH and Kim CS (2018) A review on properties of natural and synthetic based electrospun fibrous materials for bone tissue engineering. Membranes (Basel) 8, 62

35. Hasani-Sadrabadi MM, Sarrion P, Pouraghaei S et al (2020) An engineered cell-laden adhesive hydrogel promotes craniofacial bone tissue regeneration in rats. Sci Transl Med 12, 534

36. Whitehead J, Griffin $\mathrm{KH}$, Gionet-Gonzales $\mathrm{M}$, Vorwald CE, Cinque SE and Leach JK (2021) Hydrogel mechanics are a key driver of bone formation by mesenchymal stromal cell spheroids. Biomaterials 269, 120607

37. Whitehead J, Kothambawala A and Leach JK (2019) Morphogen delivery by osteoconductive nanoparticles instructs stromal cell spheroid phenotype. Adv Biosyst 3, 1900141

38. Ahmad T, ByunH, Lee J et al (2020) Stem cell spheroids incorporating fibers coated with adenosine and polydopamine as a modular building blocks for bone tissue engineering. Biomaterials 230, 119652

39. Lee W, Choi JH, Lee S, Song JE and Khang G (2020) Fabrication and characterization of silk fibroin microfiberincorporated bone marrow stem cell spheroids to promote cell-cell interaction and osteogenesis. ACS Omega 5, 18021-18027

40. Bratt-Leal AM, Carpenedo RL, Ungrin MD, Zandstra PW and McDevitt TC (2011) Incorporation of biomaterials in multicellular aggregates modulates pluripotent stem cell differentiation. Biomaterials 32, 48-56

41. Ahmad T, Shin HJ, Lee J et al (2018) Fabrication of in vitro 3D mineralized tissue by fusion of composite spheroids incorporating biomineral-coated nanofibers and human adipose-derived stem cells. Acta Biomater 74, 464-477

42. Lee J, Lee S, Ahmad T et al (2020) Human adiposederived stem cell spheroids incorporating platelet-derived growth factor (PDGF) and bio-minerals for vascularized bone tissue engineering. Biomaterials 255, 120192

43. Im Gl and Cho CS (2019) Updates in cartilage tissue regeneration. Tissue Eng Regen Med 16, 325-326

44. Martin AR, Patel JM, Zlotnick HM, Carey JL and Mauck RL (2019) Emerging therapies for cartilage regeneration in currently excluded 'red knee' populations. NPJ Regen Med 4, 12

45. Antons J, Marascio MGM, Nohava J et al (2018) Zonedependent mechanical properties of human articular cartilage obtained by indentation measurements. J Mater Sci Mater Med 29, 57

46. Prein C, Warmbold N, Farkas Z, Schieker M, Aszodi A and Clausen-Schaumann $\mathrm{H}$ (2016) Structural and mechanical properties of the proliferative zone of the developing murine growth plate cartilage assessed by atomic force microscopy. Matrix Biol 50, 1-15

47. Mansfield JC, Bell JS and Winlove CP (2015) The micromechanics of the superficial zone of articular cartilage. Osteoarthritis Cartilage 23, 1806-1816

48. Tacchetti C, Tavella S, Dozin B, Quarto R, Robino G and Cancedda R (1992) Cell condensation in chondrogenic differentiation. Exp Cell Res 200, 26-33

49. Tuli R, Tuli S, Nandi S et al (2003) Transforming growth factor-beta-mediated chondrogenesis of human mesenchymal progenitor cells involves $\mathrm{N}$-cadherin and mitogen-activated protein kinase and Wnt signaling cross-talk. J Biol Chem 278, 41227-41236

50. Jang Y, Jung H and Ju JH (2017) Chondrogenic differentiation induction of adipose-derived stem cells by centrifugal gravity. J Vis Exp 120, 54934

51. Zhang L, Su P, Xu C, Yang J, Yu W and Huang D (2010) Chondrogenic differentiation of human mesenchymal stem cells: a comparison between micromass and pellet culture systems. Biotechnol Lett 32, 1339-1346

52. Bartosh TJ, Ylöstalo JH, Mohammadipoor A et al (2010) Aggregation of human mesenchymal stromal cells (MSCs) into 3D spheroids enhances their antiinflammatory properties. Proc Natl Acad Sci U S A 107, 13724-13729

53. Yoon HH, Bhang SH, Shin JY, Shin J and Kim BS (2012) Enhanced cartilage formation via three-dimensional cell engineering of human adipose-derived stem cells. Tissue Eng Part A 18, 1949-1956 
54. Fayol D, Frasca G, Le Visage C, Gazeau F, Luciani N and Wilhelm C (2013) Use of magnetic forces to promote stem cell aggregation during differentiation, and cartilage tissue modeling. Adv Mater 25, 2611-2616

55. Zhang K, Yan S, Li G, Cui L and Yin J (2015) In-situ birth of MSCs multicellular spheroids in poly(L-glutamic acid)/ chitosan scaffold for hyaline-like cartilage regeneration. Biomaterials 71, 24-34

56. Wang W, Rigueur D and Lyons KM (2014) TGF $\beta$ signaling in cartilage development and maintenance. Birth Defects Res C Embryo Today 102, 37-51

57. Xia P, Wang X, Qu Y et al (2017) TGF-beta1-induced chondrogenesis of bone marrow mesenchymal stem cells is promoted by low-intensity pulsedultrasound through the integrin-mTOR signaling pathway. Stem Cell Res Ther 8,281

58. Yoon HH, Bhang SH, Kim T, Yu T, Hyeon T and Kim B-S (2014) Dual roles of graphene oxide in chondrogenic differentiation of adult stem cells: cell-adhesion substrate and growth factor-delivery carrier. Adv Funct Mater 24, 6455-6464

59. Kim IG, Ko J, Lee HR, Do SH and Park K (2016) Mesenchymal cells condensation-inducible mesh scaffolds for cartilage tissue engineering. Biomaterials 85, 18-29

60. Song Y, Zhang TJ, Li Y and Gao Y (2020) Mesenchymal stem cells decrease M1/M2 ratio and alleviate inflammation to improve limb ischemia in mice. Med Sci Monit 26, e923287

61. Yin C, Liang Y, Zhang J et al (2016) Umbilical cordderived mesenchymal stem cells relieve hindlimb ischemia through enhancing angiogenesis in tree shrews. Stem Cells Int 2016, 9742034

62. Yeh H-Y, Liu B-H, Sieber M and Hsu S-h (2014) Substratedependent gene regulation of self-assembled human MSC spheroids on chitosan membranes. BMC Genomics 15, 10

63. Kim S, Han YS, Lee JH and Lee SH (2018) Combination of MSC spheroids wrapped within autologous composite sheet dually protects against immune rejectionand enhances stem cell transplantation efficacy. Tissue Cell 53, 93-103

64. Park J, Choe G, Oh S and Lee JY (2020) In situ formation of proangiogenic mesenchymal stem cell spheroids in hyaluronic acid/alginate core-shell microcapsules. ACS Biomater Sci Eng 6, 6938-6948

65. Hong Y, Chen J, Fang $\mathrm{H}$ et al (2020) All-in-one hydrogel realizing adipose-derived stem cell spheroid production and in vivo injection via "Gel-Sol" transition for angiogenesis in hind limb ischemia. ACS Appl Mater Interfaces 12, 11375-11387

66. Willenborg S, Lucas T, van Loo G et al (2012) CCR2 recruits an inflammatory macrophage subpopulation critical for angiogenesis in tissue repair. Blood 120, 613-625

67. Jetten N, Donners MM, Wagenaar A et al (2013) Local delivery of polarized macrophages improves reperfusion recovery in a mouse hind limb ischemia model. PLoS One 8, e68811

68. Ran P, Chen W, Wei J et al (2020) Macrophage spheroids with chronological phenotype shifting to promote therapeutic angiogenesis in critical limb ischemia. ACS Appl Bio Mater 3, 3707-3717

69. Kieken F, Mutsaers N, Dolmatova E et al (2009) Structural and molecular mechanisms of gap junction remodeling in epicardial border zone myocytes following myocardial infarction. Circ Res 104, 1103-1112

70. Richards DJ, Tan Y, Coyle R et al (2016) Nanowires and Electrical Stimulation Synergistically Improve Functions of hiPSC Cardiac Spheroids. Nano Lett 16, 4670-4678

71. Tan Y, Richards D, Xu R et al (2015) Silicon nanowireinduced maturation of cardiomyocytes derived from human induced pluripotent stem cells. Nano Lett 15, 2765-2772

72. Park J, Kim YS, Ryu S et al (2015) Graphene potentiates the myocardial repair efficacy of mesenchymal stem cells by stimulating the expression of angiogenic growth factors and gap junction protein. Adv Funct Mater 25, 2590-2600

73. DiMeglio LA, Evans-Molina C and Oram RA (2018) Type 1 diabetes. The Lancet 391, 2449-2462

74. Akolpoglu MB, Inceoglu Y, Bozuyuk $U$ et al (2021) Recent advances in the design of implantable insulin secreting heterocellular islet organoids. Biomaterials 269, 120627

75. Vantyghem M-C, de Koning EJP, Pattou F and Rickels MR (2019) Advances in $\beta$-cell replacement therapy for the treatment of type 1 diabetes. The Lancet 394, 1274-1285

76. Foster GA and Garcia AJ (2017) Bio-synthetic materials for immunomodulation of islet transplants. Adv Drug Deliv Rev 114, 266-271

77. Llacua LA, Faas MM and de Vos P (2018) Extracellular matrix molecules and their potential contribution to the function of transplanted pancreatic islets. Diabetologia 61, 1261-1272

78. Bochenek MA, Veiseh O, Vegas AJ et al (2018) Alginate encapsulation as long-term immune protection of allogeneic pancreatic islet cells transplanted into the omental bursa of macaques. Nat Biomed Eng 2, 810-821

79. Coronel MM, Martin KE, Hunckler MD et al (2020) Immunotherapy via PD-L1-presenting biomaterials leads to long-term islet graft survival. Sci Adv 6, eaba5573

80. Yang J, Jiang S, Guan $Y$ et al (2019) Pancreatic islet surface engineering with a starPEG-chondroitin sulfate nanocoating. Biomater Sci 7, 2308-2316

81. Headen DM, Woodward KB, Coronel MM et al (2018) Local immunomodulation Fas ligand-engineered biomaterials achieves allogeneic islet graft acceptance. Nat Mater 17, 732-739

82. Liu JMH, Zhang J, Zhang $X$ et al (2016) Transforming growth factor-beta 1 delivery from microporous scaffolds decreases inflammation post-implant and enhances function of transplanted islets. Biomaterials 80, 11-19

83. Jiang K, Chaimov D, Patel SN et al (2019) 3-D physiomimetic extracellular matrix hydrogels provide a supportive microenvironment for rodent and human islet culture. Biomaterials 198, 37-48

84. Daoud J, Petropavlovskaia M, Rosenberg L and Tabrizian $M$ (2010) The effect of extracellular matrix components on the preservation of human islet function in vitro. Biomaterials 31, 1676-1682

85. Goh SK, Bertera S, Olsen P et al (2013) Perfusiondecellularized pancreas as a natural 3D scaffold for pancreatic tissue and whole organ engineering. Biomaterials 34, 6760-6772

86. Lee KY and Mooney DJ (2012) Alginate: properties and 
biomedical applications. Prog Polym Sci 37, 106-126

87. Radu RG, Fujimoto S, Mukai E et al (2005) Tacrolimus suppresses glucose-induced insulin release from pancreatic islets by reducing glucokinase activity. Am J Physiol Endocrinol Metab 288, E365-371

88. Ji S, Zhu Z, Sun X and Fu X (2021) Functional hair follicle regeneration: an updated review. Signal Transduct Target Ther 6, 66

89. Stenn KS and Paus R (2001) Controls of hair follicle cycling. Physiol Rev 81, 449-494

90. Rahmani W, Abbasi S, Hagner A et al (2014) Hair follicle dermal stem cells regenerate the dermal sheath, repopulate the dermal papilla, and modulate hair type. Dev Cell 31, 543-558

91. Huang YC, Chan CC, Lin WT et al (2013) Scalable production of controllable dermal papilla spheroids on PVA surfaces and the effects of spheroid size on hair follicle regeneration. Biomaterials 34, 442-451

92. Zhang K, Bai X, Yuan Z et al (2020) Cellular nanofiber structure with secretory activity-promoting characteristics for multicellular spheroid formation and hair follicle regeneration. ACS Appl Mater Interfaces 12, 7931-7941

93. Wang J, Miao Y, Huang Y et al (2018) Bottom-up nanoencapsulation from single cells to tunable and scalable cellular spheroids for hair follicle regeneration. Adv Healthc Mater 7, 1700447

94. Wong VW, Akaishi S, Longaker MT and Gurtner GC (2011) Pushing back: wound mechanotransduction in repair and regeneration. J Invest Dermatol 131, 2186-2196

95. Vining KH and Mooney DJ (2017) Mechanical forces direct stem cell behaviour in development and regeneration. Nat Rev Mol Cell Biol 18, 728-742

96. Nowell CS, Odermatt PD, Azzolin L et al (2016) Chronic inflammation imposes aberrant cell fate in regenerating epithelia through mechanotransduction. Nat Cell Biol 18, 168-180

97. Meli VS, Atcha H, Veerasubramanian PK et al (2020) YAP-mediated mechanotransduction tunes the macrophage inflammatory response. Sci Adv 6, eabb8471 